\title{
Validation of the Vertical Heterophoria Symptom Questionnaire (VHS-Q) In Patients with Balance Problems and Binocular Visual Dysfunction after Acquired Brain Injury
}

\author{
Trine Schow ${ }^{1 *}$, Thomas William Teasdale ${ }^{2}$ and Morten Arendt Rasmussen ${ }^{3}$ \\ ${ }^{1}$ Brain Injury Center - BOMI, Roskilde, Denmark \\ ${ }^{2}$ Department of Psychology, University of Copenhagen. Denmark \\ ${ }^{3}$ Faculty of Science, Spectroscopy and Chemometrics, University of Copenhagen. Denmark
}

Received: March 18, 2016; Accepted: April 26, 2016; Published: May 06, 2016

*Corresponding author: Trine Schow, Brain Injury Center - BOMI, Maglegaardsvej 15, 4000 Roskilde, Denmark, Tel: +45 3-084-4481; E-mail: trineschow@bomi.roskilde.dk

\begin{abstract}
Objectives: To evaluate the utility of the Vertical Heterophoria Symptom Questionnaire (VHS-Q) in subjects with stroke and problems with Ocular Motility Dysfunction (OMD) and balance. study.

Design: Our data were derived from a longitudinal intervention

Method: Subjects completed a 12-week intervention program with OMD rehabilitation and balance training. They were tested with the VHS-Q, and objective OMD measures, at baseline, at the end of the intervention period and at three- and six-month follow-ups. We performed a Principal Component Analysis with orthogonal rotation to extract underlying factors from the VHS-Q. The association between these factors and patient background information was analyzed using a repeated measures analysis of variance. Associations between the VHS-Q factors and objective OMD measurements were assessed using pair wise correlation analysis.
\end{abstract}

Participants: 29 adults with stroke aged 18-67 years.

Results: Four components were extracted from the VHS-Q, explaining $55 \%$ of item variation: Dizziness, Reading, Pain and Visual function. Convergent validity was established with objective OMD measures. Gender was a strong determinant for visual impairment affecting "reading" and "pain". Treatment time was related with visual impairment.

Conclusion: The VHS-Q can be used to evaluate the impact of OMD in a stroke population. The psychometric constructs make it useful for evaluating OMD, planning intervention and improving future research.

Keywords: Multivariate analysis; Stroke; Ocular Motility Dysfunction

\section{Introduction}

Visual impairment following stroke is very common $[9,7,22]$ and can involve sensory, motor and perceptual dysfuntioning
[21]. It is, however, a rather neglected topic in neuro rehabilitation research. Ocular motility dysfunction (OMD) has been reported in $90 \%$ in patients with brain injury $[10,12]$. The problems are manifold and may involve difficulties with reading, working at computers, using smart phones, driving a car etc. This means that the patient's activities of daily living, mobility, and participation in rehabilitation programs are affected and these in turn represent a significant barrier to returning to work.

The association with OMD and other symptoms has, however, not been clearly established, and solid scientific research demonstrating the efficacy of visual rehabilitation has been limited [2]. In recent years, there has been a growing body of evidence showing positive results from optometric visual therapy [4]. Both subjective and objective measures are important in guiding the best possible rehabilitation program. Subjective symptoms are often the first that the patient becomes aware of and they are what encourages the patient to go to a health-care professional and thereby indicate to the professional that something is wrong. Subjective symptom questionnaires are therefore warranted. However, there are as yet no standardized measures in the area of vision disturbance for the adult stroke population [7].

The College of Optometrists in Vision Development Quality of Life checklist (COVD) [17] is the most frequently used questionnaire survey, and was recently chosen as part of a vision screening for patients with tramatic brain injury (TBI) [20]. The COVD covers symptoms associated with vision disturbances in four broad aspects of quality of life, being a non-diagnosis specific questionnaire. It has been demonstrated to effectively detect changes in symptoms in children and students $[15,16]$. By contrast, [6] identified a set of symptoms typical for patients that are associated with vertical heterophoria $(\mathrm{VH})$ (in which one eye is higher than the other eye), a form of binocular dysfunction, and 
significantly overlapping symptoms after traumatic brain injury (TBI) including pain, head tilt, dizziness, reading difficulties, routine visual, binocular visual symptoms and psychological symptoms. From these seven symptom categories, the authors have developed a self-administered survey instrument, namely the Vertical Heterophoria Symptom Questionnaire (VHS-Q) comprising 25 questions. In our rehabilitation center, patients with balance problems and binocular visual dysfunction after stroke are treated by a team including two optometrists and two physiotherapists. We found that our patients' symptoms were similar to those covered by the VHS-Q, whereas the questions in the COVD concerning school work and leisure sports activities were less relevant and there is a lack of questions regarding dizziness, which is common among our patients. Therefore the VHS-Q was chosen as a screening tool in this study investigating patients with balance and OMD.

The objective of the study was to statistically evaluate the utility of the VHS-Q in a group of stroke subjects with OMD, using objective binocular vision measures to assess convergent validity. Moreover, we aimed to investigate the distribution of the 25 VHS-Q questions in relation to underlying symptom categories using Principal Component Analysis (PCA) with Varimax rotation.

\section{Methods}

Data for this study were collected both pre- and post an intervention period for four month and six months. The study was approved by the Danish Data Protection Agency j.nr. 2014-41-3324. Participants were recruited from 22 different municipalities in Denmark and from three regional hospitals. The participants received intensive training, in groups of six or seven over a four-month period, by an optometrist and physiotherapists specialized in OMD and balance problems [27].

\section{Inclusion criteria}

Subjects with a) stroke (within 3 to 36 months prior to program entry), b) balance and oculomotor problems and c) age between 18 and 67 years.

\section{Exclusion criteria}

Previous history of OMD and balance problems, hemianopia and any other OMD, progressive and/or severe eye disease, degenerative brain disease, severe cognitive problems to an extent that participation in group training would not be possible, a psychiatric diagnosis or drug abuse.

Thirty-three subjects were referred to the study. Three subjects did not fulfill the inclusion criteria and one was later excluded due to hospitalization. The remaining 29 agreed to participate and signed an informed consent form.

\section{Outcome measures}

The VHS-Q was designed to assess the severity of symptoms and to assess improvement of symptoms with treatment. Questions cover seven major symptom categories: head and eye pain, dizziness, reading difficulties, binocular vision symptoms, routine vision symptoms, and anxiety. It consists of 25 questions. For each question the patient is asked to mark the answers that best describes his/her situation with a score of $0-3$, where $0=$ never; 1 = occasionally; 2 = frequently and $3=$ always [6].

The VHS-Q scale was translated into Danish following recommended procedures [23]. First, the scale was translated into Danish by two health-science researchers both having Danish as their mother tongue whilst being fluent in English. They agreed on one Danish version, which was back-translated into English by another researcher fluent in Danish but having English as her mother tongue. Mark Rosner, one of the original developers of the VHS-Q [6] has approved the translation on the basis of the back-translation.

Objective OMD Test: Stereopsis at near distance (SAN) was assessed using the Randot Stereotest (Stereo Optical Co, Chicago, IL) with subjects wearing Polaroid spectacles. The test stereogram was held at a distance of $40 \mathrm{~cm}$ from the subject during testing. Patients with refractive errors wore their spectacles under their Polaroid lenses. Patients were asked to determine which circle in each successive group appeared to "pop out of the page". This procedure was repeated until two mistakes were made successively. Threshold stereo acuity level was recorded in seconds of arc.

A Reading-related saccadic eye movement was measured using the King-Devick test (K-D). This test involves reading aloud a series of randomized single numbers from left to right as quickly as possible. The numbers are separated by varying spacing, either with or without a connecting line. The K-D test includes one demonstration card and three test cards that increase in difficulty. Grading for the K-D is based on the time taken to read 40 numbers aloud.

Near-point of convergence (NPC) was assessed using a Wolff Wand placed along the subject's visual midline in primary position at $57 \mathrm{~cm}$. Then the Wand was slowly moved towards the bridge of the nose. A break of NPC was recorded either when the patient reported diplopia or when the examiner observed one eye turning away. Then the Wand was moved away from the bridge of the nose, and the patient was asked when fusional recovery occurred (Recovery) [3].

Positive and negative relative vergence were done both at far distance $=6 \mathrm{~m}$ (PRVD and NRVD) and at near distance $=40 \mathrm{~cm}$ (PRVN and NRVN) following a standard procedure by using basein and base-out prisms, respectively [8].

Binocular fusion was tested using the Keystone ophthalmic tele binocular utilizing the Keystone Visual Skills Series tests both at far distance (BFD) and near distance (BFN). We used tests DB-4K and DB-5K(Keystone).

\section{Statistical analysis}

Extraction of factors from VHS-Q data: The 25 questions in the VHS-Q survey reveal several underlying constructs, or factors, related to different parts of vision functionality. In order to extract these underlying factors a principal component analysis (PCA) with varimax rotation was used to derive a set of factors that reflect uncorrelated functionality patterns based on the VHS-Q survey data from pre-intervention (0 month), during 
intervention (two and four months) and three and six month post intervention ( 7 and 10 month). The number of factors retained in the model was determined by examination of scree plots of eigen values and the interpretability of the rotated factor loadings They were then further validated by a non-parametric bootstrap procedure (with 10,000 iterations), determining the factor loading uncertainty. In addition to these data-derived factors, a total score (the sum of responses to all 25 questions) was calculated. This additional factor is not independent of the PCA factors and is merely a more global representation of the VHS-Q data.

Association with patient background characteristics: The association between VHS-Q factors and the independent variables; gender, age, time since injury, Injury localization and home-exercises were analyzed within a repeated measures analysis of variance, with the independent variables and time from onset of treatment as categorical predictors, including the interaction of these and the individual VHS-Q factors as responses. Age and Home-exercises were treated as categorical variables divided into tertiles.

Association with objective symptom characteristics: The association between VHS-Q factors and the objective symptom characteristics (SAN, K-D, NPC, PRVN, NRVN, PRVD, NRVD and $B F N, B F D$ ) were examined with partial correlation analysis, where the overall effect of time and patient differences were removed, i.e. correcting the analysis for the overall effect of treatment duration (time) and differences between patients due to age, gender, etc., and hence focusing on the common dynamics between objective and subjective symptom characteristics.
The PCA was conducted using the PLS toolbox (eigenvector inc () , and pair wise correlations were calculated using the builtin correlation function both within Matlab® R2014b. Repeated measures models were conducted by the GLS function in the statistical package NIME (ver. 3.1-117) for R 3.1.1. Spider plots were produced by the routine downloaded from www.models. life.ku.dk/dietarypattern; the remaining plotting was conducted by in-house routines.

\section{Results}

All 29 participants completed the training period. Follow-up data were excluded from further analysis for three participants who suffered from recurrent stroke. At baseline there were 13 (45\%) women. For the genders together, the mean age was 52.9 years (SD: 7.5). The localization of the stroke was as follows: brainstem $=14 \%$; cerebellum $=24 \%$; Right hemisphere $=10 \%$; Left hemisphere $=28 \%$ and bilateral hemisphere $=24 \%$.

\section{VHS-Q factor structure, item analysis and convergent validity}

We used a PCA with varimax rotation to identify subscales from the VHS-Q 25 items and to establish whether they structured according to the symptom-type categories which underlie the scales. Based on the eigenvalue scree plot) and interpretability of the factor loadings (see Figure 1), four factors were extracted. A five-component solution revealed four similar patterns and a non-interpretable fifth; the four were retained. Based on the question types these factors were identified as "dizziness" (component 1 - 23\% variance explained), "reading"
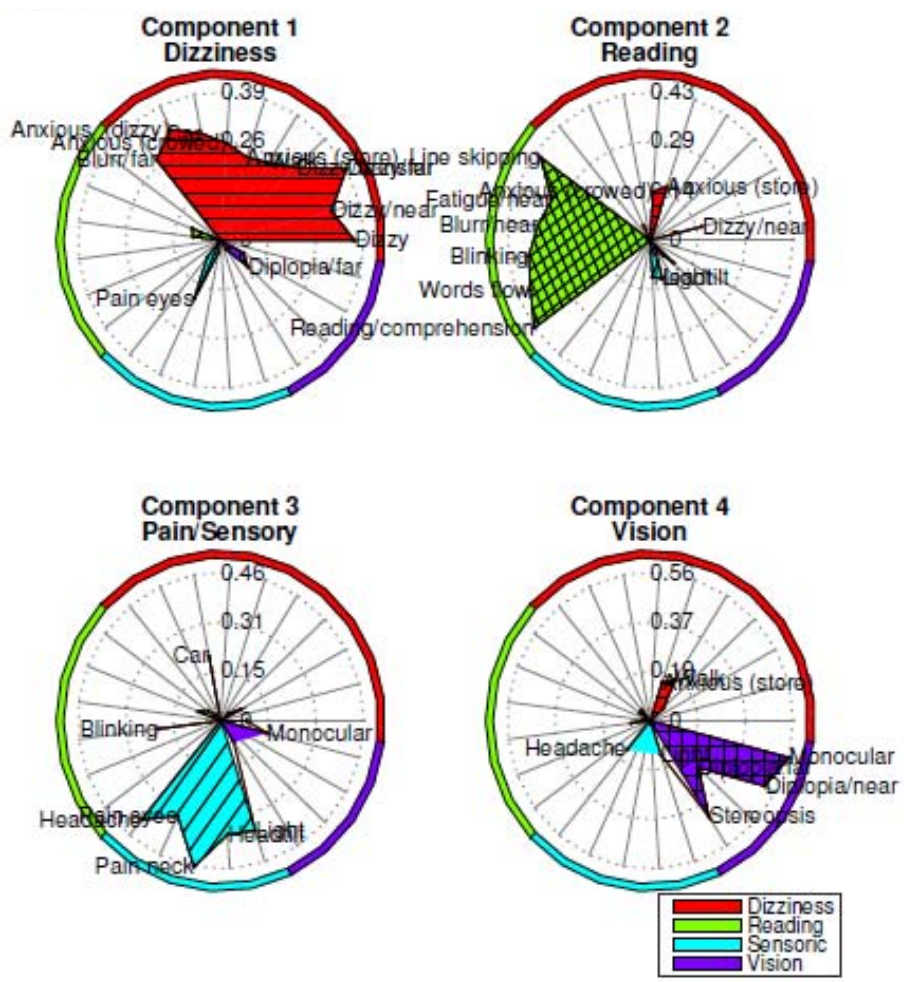

Figure 1: Spider plot of the four factor loadings. 
related problems (component 2 - 17\% variance explained), "pain/sensory" issues (component 3 - 11\% variance explained) and "vision" disturbance such as double vision and stereopsis (component $4-6 \%$ variance explained). The bootstrap analysis showed high internal consistency for all four components with respect to item uncertainty, where especially component 1 "dizziness" and 2 "reading" were shown to be very robust constructs (see Figure 2).

Table 1 shows the relationship between the VHS-Q factors and patient background variables, where female gender is the strongest predictor of visual impairment affecting both components 2 and 3 and consequently also the total score, meaning that women have higher levels of reading problems and pain/sensory problems than men. The strongest, and probably most trivial, determinant for visual impairment is treatment time; however, component 3 only shows little development over time. Injury localization is significantly associated with component 1 , where in particular patients with a cerebellar injury and bilateral injury and to some extent left hemisphere injuries obtain higher scores than patients with a brain stem or a right side injury. Finally being older ( $>57$ years) is associated with a higher score on component 4 .

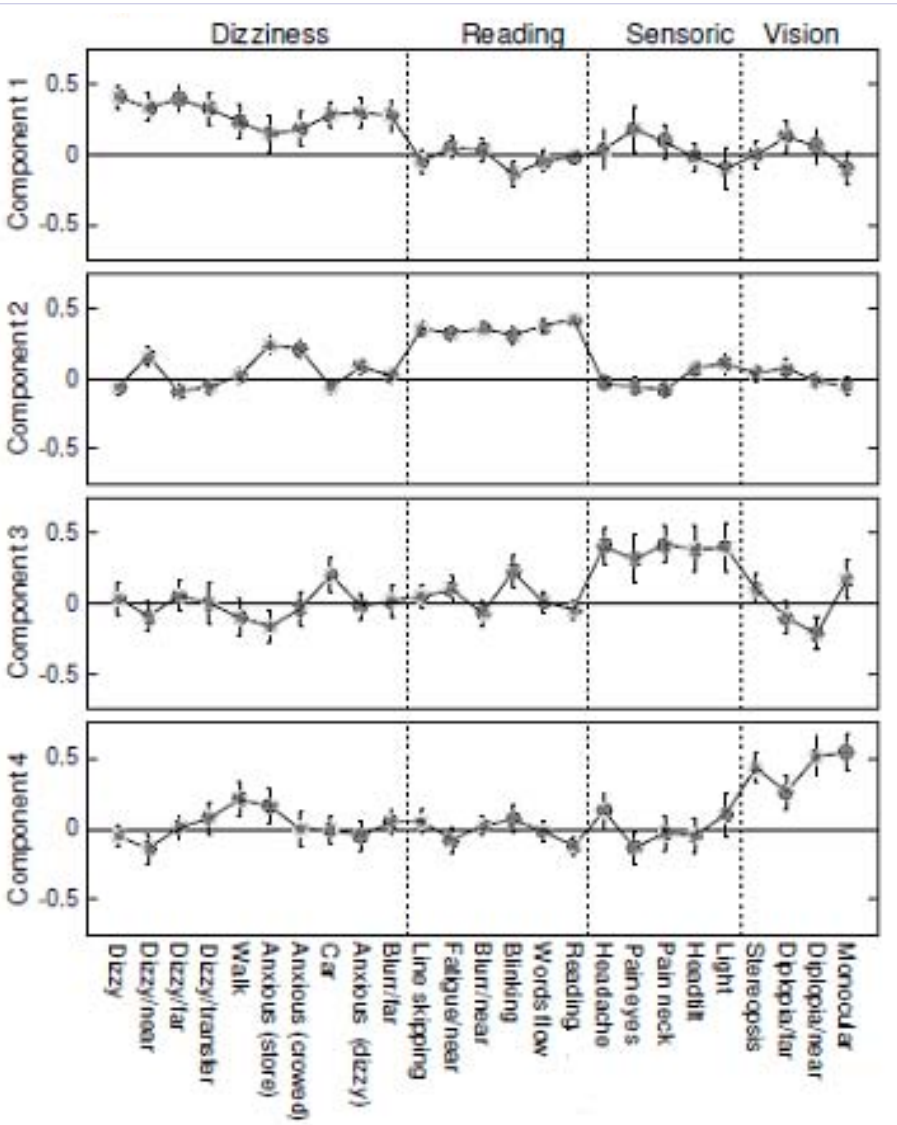

Figure 2: Uncertainty measures for factor loadings (Bootstrap results).

Table 1: Association between patient characteristics and VHS-Q factors.

\begin{tabular}{|c|c|c|c|c|c|c|c|c|c|c|c|}
\hline p-value & Time & $\begin{array}{c}\text { Injury } \\
\text { localization }\end{array}$ & $\begin{array}{c}\text { Injury } \\
\text { localization } x \text { time }\end{array}$ & TSI & TSI $x$ time & age & age $x$ time & \begin{tabular}{|c|} 
Home \\
exercises
\end{tabular} & \begin{tabular}{|c|} 
Home \\
exercises time
\end{tabular} & Sex & Sex $x$ time \\
\hline $\begin{array}{c}\text { Component } \\
1\end{array}$ & $<0.001$ & 0.017 & 0.321 & 0.338 & 0.901 & 0.875 & 0.127 & 0.370 & 0.507 & 0.099 & 0.948 \\
\hline $\begin{array}{c}\text { Component } \\
2\end{array}$ & $<0.001$ & 0.447 & 0.883 & 0.692 & 0.075 & 0.301 & 0.533 & 0.091 & 0.903 & $<0.001$ & 0.915 \\
\hline $\begin{array}{c}\text { Component } \\
3\end{array}$ & 0.083 & 0.085 & 0.158 & 0.102 & 0.796 & 0.482 & 0.223 & 0.435 & 0.868 & $<0.001$ & 0.612 \\
\hline $\begin{array}{c}\text { Component } \\
4\end{array}$ & 0.003 & 0.373 & 0.961 & 0.217 & 0.917 & 0.003 & 0.137 & 0.883 & 0.645 & 0.500 & 0.398 \\
\hline Total & $<0.001$ & 0.035 & 0.352 & 0.719 & 0.367 & 0.709 & 0.709 & 0.502 & 0.999 & 0.277 & 0.598 \\
\hline
\end{tabular}

All numbers represent $\mathrm{p}$-values. Statistical significant $\mathrm{p}<0.05$ marked in bold. 


\section{Objective measures}

The associations between the VHS-Q factors and the objective symptom measures shows that NRVD is related to component 1 (dizziness), KD, NPC and NRVN and PRVN to component 2 (reading), BFN, PRVN and NRVN to component 3 (pain/sensory) and K-D, BFN, PRVN, NRVD and PRVD to component 4 (vision) (see Figure 3).

\section{Discussion}

The VHS-Q was used to investigate the burden of ocular motor dysfunction in a sample of patients with acquired brain injury participating in an intervention study. In this paper we have presented the results from what we believe to be the first investigation of the utility of the Danish translation of the VHS-Q.

Using PCA, we found that the VHS-Q loaded on four components and not six symptoms categories as used by [6]. However, our four components largely covered the six categories as originally described: 1) Reading problems, 2) Dizziness, 3) Pain, 4) Binocular Vision problems explaining in total $56 \%$ of the item variance.

Question (Q) 1, 2 and 3 of pain and neck discomfort loaded together with item 13 "head tilt" and item 17 "bright light sensitivity" on component 3 . Head tilt might cause neck pain and therefore load with pain questions, just as light sensitivity may cause pain in eyes or head, explaining this coherence $[24,19,26]$.

Q19-20 and Q22-25 all relate to reading and close up activities and therefore correlate very well with the "reading" category described by original author [6] , just as Q14-16 and Q18 load on the category "binocular vision" and these questions all concerns double vision and depth perception.

Q4 to Q12 loaded on component 2 "dizziness". These questions involve the major category of psychological symptoms described by [6], where subjects feel anxiety due to dizziness and being in a crowd and also questions concerning the feeling of dizziness. Dizziness may result in anxiety, due to the risk of falling; however, adults with anxiety disorders are also likely to experience dizziness [25]. Therefore, this phenomenon may be a 'two-way street'. Since the subjects in this study did not suffer from a psychiatric illness, it is most likely that it is the risk of falling that result in anxiety rather than the reverse. However, it may be relevant to discuss other options if treatment fails. Item 21 blurred vision at distance also loaded on dizziness. Patients who experience blurred vision with far-vision activities might feel dizzy since it becomes difficult to orientate in relation to others [7], as compared with blurred vision in close-up activities.

We correlated the four factor components with the objective visual measures used in the intervention study. Component 2 - reading problems - had strong correlations with KD and NPC and PRVN which all represent movements needed in reading, suggesting a strong convergent validity of this component. NRVD and PRVD and PRVN and BFN all correlated with vision. These measures of fusional vergence are all associated with keeping binocular vision [5]. However, we had expected that SAN would prove to correlate with this item, since it is highly correlated with item 14 "do you experience poor depth perception", but this did not emerge in the analyses. NRVD is the only measure correlating with the component dizziness. NRVD is involved when screening surroundings, walking around in a crowd or a store, and reported

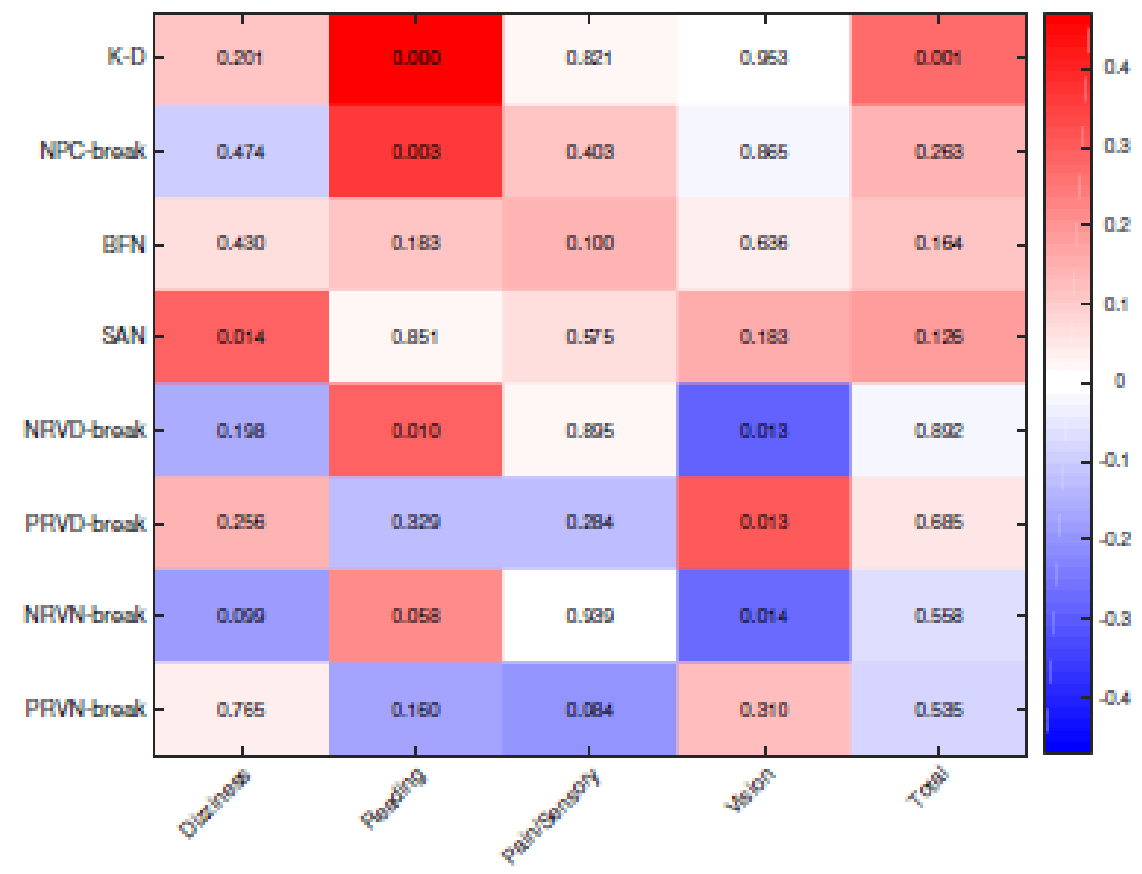

Figure 3: Association plot between factor scores and objective measures (heat map). The color represent correlation values, numbers are p-values. 
dizziness might be a symptom of negative fusional vergence. BFN, PRVN and NRVN are correlated to the third component, namely pain. There is a growing body of research showing that an increase in visual load (activity levels of eye muscles) over time gives rise to a parallel increase in musculoskeletal load and symptoms in the neck/scapular area [26]. Patients with fusional vergence problems may have an extraocular muscle strain eventually leading to ocular muscle fatigue and thereby pain in the neck area [18].

We further looked at the association between VHS-Q factors and the demographic and medical information; gender, age, time since injury, injury localization and home-exercises. These analyses should be viewed with caution in view of the low number of participants. However, we found that participants with a cerebellar injury had a higher score on component 1 : dizziness, which is in line with the fact that the cerebellum plays a crucial role in dizziness and balance [14].

That women experience higher pain (component 3) than men concurs well with a review by who investigated a wide variety of painful conditions [13]. The review found gender differences in pain perception, and clinical studies clearly demonstrate that women are at greater risk for chronic pain than are men [13]. Furthermore, we found that greater age is correlated with component 4-vision which is in line with the consistent evidence that visual problems worsen with age [1]. This appeared in our data despite our avoiding the most severe age-related bias by setting the maximum age for participants in our study to 67 years.

The VHS-Q was developed originally in relation to the symptom burden of patients with TBI and vertical heterophoria symptoms; however, we found the questionnaire to be highly relevant in a stroke population, which also includes other forms of oculomotor difficulties. Our findings revealed that the VHS-Q reflects four subscales that correlate in a relevant and meaningful way with objective measures and with demographic and medical characteristics. This nuanced information is partly lost when using only the combined information of a single total score. The subscales represent significant clinical information of relevance when planning intervention and additional measures. We believe that it will be very worthwhile to conduct further evaluations and validations of this questionnaire in larger-scale studies.

\section{Limitations}

This study was a prospective longitudinal study design, which by its nature presents difficulties in patient recruitment. For this reason we had only a relatively small sample of subjects resulting in low statistical power.

\section{Conclusion}

This study is, to our knowledge, the first attempt to investigate the utility of the Danish VHS-Q. The study demonstrates that the VHS-Q is a valid multidimensional measure that can be used to evaluate the impact of oculomotor problems in stroke patients or problems with dizziness, reading, pain and visual function. We have found this instrument to be highly relevant as a measure of symptom burden and intervention planning in stroke patients.

\section{Acknowledgment}

We thank the patients for participating in this study, the professionals in the intervention team and external testers for their contribution to this study's development and process. We wish also to extend a special thanks to the Danish Brain Injury Association and the Danish Stroke Association for their support.

This study was carried out with financial support from the Fund for Better Working Environment and Labour Retention and the Danish Health Foundation. The authors have no conflict of interests in connection with this study.

\section{References}

1. Akpek EK and RA Smith.Overview of age-related ocular conditions. Am J Manag Care. 2013;(19):S67-75.

2. Barrett B. A critical evaluation of the evidence supporting the practice of behavioural vision therapy. Ophthalmic and physiological optics. 2009;29(1):4-25. doi: 10.1111/j.1475-1313.2008.00607.x.

3. Ciuffreda KJ and DP Ludlam . Objective diagnostic and interventional vision test protocol for the mild traumatic brain injury population. Optometry. 2011;82(6):337-339. doi: 10.1016/j.optm.2011.03.006.

4. Ciuffreda KJ, D Rutner, N Kapoor, IB Suchoff, S Craig and ME Han. Vision therapy for oculomotor dysfunctions in acquired brain injury: a retrospective analysis. Optometry. 2008;79(1):18-22.

5. Cooper J. Clinical implications of vergence adaptation.Optom Vis Sci 1992;69(4):300-307.

6. Doble JE, DL Feinberg, MS Rosner and AJ Rosner. Identification of binocular vision dysfunction (vertical heterophoria) in traumatic brain injury patients and effects of individualized prismatic spectacle lenses in the treatment of postconcussive symptoms: a retrospective analysis. PM R. 2010;2(4):244-253.

7. Greenwald BD, N Kapoor and AD Singh. Visual impairments in the first year after traumatic brain injury. Brain Inj. 2012;26(11): 1338-1359. doi: 10.3109/02699052.2012.706356.

8. Grosvenor T. Primary Care Optometry. Philadelphia, Elsevier Health Sciences. 2006

9. Jones SA and RA Shinton. Improving outcome in stroke patients with visual problems. Age Ageing. 2006;35(6):560-565.

10. Kapoor N, KJ Ciuffreda and Y Han.Oculomotor rehabilitation in acquired brain injury: a case series. Arch Phys Med Rehabil 2004;85(10):1667-1678.

11. Keystone Mast Concepts. Keystone View. Nevada Capital Group Inc., 2200 Dickerson Road, Reno, NV 89503.

12. Khan S, E Leung and WM Jay. Stroke and visual rehabilitation. Top Stroke Rehabil. 2008;15(1):27-36. doi: 10.1310/tsr1501-27.

13. Kvachadze I, MG Tsagareli and Z Dumbadze. An overview of ethnic and gender differences in pain sensation. Georgian Med News. 2015;(238):102-108.

14. Manto M-UM and M Pandolfo. The Cerebellum and Its Disorders. United Kingdom, Cambridge. 2001. doi.org/10.1017/CBO9780511666469

15. Maples WC. Test-retest reliability of the College of Optometrists in Vision Development Quality of Life Outcomes Assessment. Optometry. 2000;71(9):579-585.

16. Maples WC and M Bither. Efficacy of vision therapy as assessed by the 
COVD quality of life checklist. Optometry 2002;73(8): 492-498.

17. Mozlin R. Quality of life outcomes assessment. J Optom Vis Dev 1995;26:194-199.

18. Neugebauer A, J Fricke and W Russmann. Asthenopia: frequency and objective findings. Ger J Ophthalmol 1992;1(2):122-124.

19. Noseda R. Unanswered questions in headache: so what is photophobia, anyway? Headache. 2013;53(10):1679-1680. DOI: 10.1111/ head. 12230

20. Radomski MV, M Finkelstein, I Llanos, M Scheiman and SG Wagener . Composition of a vision screen for service members with traumatic brain injury: consensus using a modified nominal group technique. Am J Occup Ther. 2014;68(4):422-429. doi: 10.5014/ajot.2014.011445.

21. Rowe F. Symptoms of stroke-related visual impairment. Strabismus 2013;21(2):150-154. doi: 10.3109/09273972.2013.786742.

22. Rowe F, Wright D, Brand D, Jackson A, Price L, Walker S, et al. Reading difficulty after stroke: ocular and non ocular causes. Int J Stroke. 2011;6(5):404-411. doi: 10.1111/j.1747-4949.2011.00583.x.

23. Sechrest L, Tl Fay and SMH Zaidi . Problems of translation in cross-cultural research. J Cross Cult Res. 1972;3(1):41-56. doi: $10.1177 / 002202217200300103$

24. Shapiro IJ. Relation between vertical facial asymmetry and postural changes of the spine and ancillary muscles. Optom Vis Sci. 1994;71(8):529-538.

25. Staab JP. The influence of anxiety on ocular motor control and gaze. Curr Opin Neurol. 2014;27(1):118-124. doi: 10.1097/ WC0.0000000000000055.

26. Zetterberg C, M Forsman and HO Richter. Effects of visually demanding near work on trapezius muscle activity. I Electromyogr Kinesiol. 2013;23(5):1190-1198. doi: 10.1016/j.jelekin.2013.06.003.

27. Schow T, Harris P, Teasdale TW and Rasmussen MA. Evaluation of a four month rehabilitation program for stroke patients with balance problems and binocular visual dysfunction. NeuroRehabilitation 2016. 